\title{
DỨBin
}

Technological University Dublin ARROW@TU Dublin

2004-01-01

\section{Torsion-Free Weakly Transitive Abelian Groups}

\author{
Brendan Goldsmith \\ Technological University Dublin, brendan.goldsmith@tudublin.ie \\ Lutz Strungmann \\ Universitat Duisberg Essen
}

Follow this and additional works at: https://arrow.tudublin.ie/scschmatart

Part of the Mathematics Commons

\section{Recommended Citation}

Goldsmith, B. \& Strungmann, L. (2004). Torsion-free weakly transitive Abelian groups. Communications in Algebra, vol. 33, no. 4, pg. 1177 - 1191. doi:10.1081/AGB-200053836

This Article is brought to you for free and open access by the School of Mathematics at ARROW@TU Dublin. It has been accepted for inclusion in Articles by an authorized administrator of ARROW@TU Dublin. For more information, please contact arrow.admin@tudublin.ie, aisling.coyne@tudublin.ie,gerard.connolly@tudublin.ie.

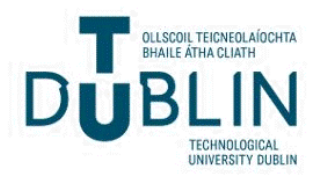




\title{
Torsion-Free Weakly Transitive Abelian Groups
}

\author{
Brendan Goldsmith and Lutz Strüngmann * \\ School of Mathematics and Sciences, Dublin Institute of Technology, \\ Dublin, Ireland \\ Fachbereich 6, Mathematik, Universität Duisburg-Essen, \\ Germany
}

\begin{abstract}
We introduce the notion of weak transitivity for torsion-free abelian groups. A torsion-free abelian group $G$ is called weakly transitive if for any pair of elements $x, y \in G$ and endomorphisms $\varphi, \psi \in \operatorname{End}(G)$ such that $x \varphi=y, y \psi=x$ there exists an automorphism of $G$ mapping $x$ onto $y$. It is shown that every suitable ring can be realized as the endomorphism ring of a weakly transitive torsion-free abelian group and we characterize up to a number theoretical property the separable weakly transitive torsion-free abelian groups.
\end{abstract}

\section{INTRODUCTION}

The notions of transitivity and full transitivity were introduced in 1954 by Kaplansky (1969) in the context of his study of abelian $p$-groups or, equivalently, in the context of primary modules over a complete discrete valuation ring. Since then the notions have been extensively studied and the concepts have been widened to include torsion-free and mixed abelian groups and modules see e.g. Carroll and Goldsmith (1996), Corner (1976), Files and Goldsmith (1998), Grinshpon (1982), Hennecke (1999), Hill (1969), Megibben (1966), Paras and Strüngmann (2003) for the torsion case, Files (1996, 1997), Hennecke and Strüngmann (2000) for the mixed case and Dobrusin (1985), Dugas and Shelah (1989), Grinshpon (1982),

Mathematics Subject Classification (2000): 20K01, 20K10, 20 K30.

*Supported by the Deutsche Forschungs Gesellschaft. The authors would like to thank Dani for her beautiful typing of this manuscript. 
Grinshpon and Misyakov (1986, 1991), Hausen (1987) and Krylov (1986, 1989) for the torsion-free case. Recall that an abelian $p$-group $G$ is said to be (fully) transitive if for any $x, y \in G$ with $\left(U_{G}(x) \leq U_{G}(y)\right) U_{G}(x)=U_{G}(y)$ there exists an (endomorphism) automorphism of $G$ mapping $x$ onto $y$. Here the Ulm sequence $U_{G}(z)$, for any $z \in G$, is the sequence of heights $h t^{G}(z), h t^{G}(p z), \cdots$ and such sequences are ordered pointwise - see Kaplansky (1969) for further details. A similar definition holds for a torsion-free abelian group $G$ with the Ulm sequences in the definition being replaced by the corresponding height sequences $h t_{p_{1}}^{G}(z), h t_{p_{2}}^{G}(z), \cdots$ where $\left\{p_{n}: n \in \mathbb{N}\right\}$ is the set of primes in their natural order. It will be convenient to continue to denote the height sequence in this torsion-free case by $U_{G}(x)$. Generalizing these transitivity concepts to arbitrary abelian groups is not easy since the concepts are phrased in terms of elements and indicator sequences (height sequences); note that Files $(1996,1997)$ has introduced some generalizations for local mixed groups. In the present work we introduce a transitivity-type concept which avoids reference to indicator sequences: we say that an abelian group $G$ is weakly transitive if for any pair of elements $x, y \in G$ and endomorphisms $\varphi, \psi$ of $G$ such that $x \varphi=y$ and $y \psi=x$ there exists an automorphism of $G$ mapping $x$ onto $y$. After some observations about mixed abelian groups we focus on the torsion-free situation and show that the class of weakly transitive abelian groups is extensive and coincides with neither the class of transitive nor the class of fully transitive abelian groups. In particular we consider separable torsion-free abelian groups and among those characterize the weakly transitive ones up to some number theoretical problem. In addition to the advantage of having a concept of transitivity which is "indicator-sequence free", the simple fact that a fully transitive group is transitive if and only if it is weakly transitive makes this latter concept a natural object to study. Moreover, in the $P$-local case, where $P$ is a finite set of primes, transitivity implies full transitivity, hence weak transitivity is exactly the missing property that distinguishes the $P$-local transitive torsion-free groups from the fully transitive ones.

Henceforth all references to a "group" shall mean an additively written abelian group; automorphism groups which will always be written multiplicatively may, of course, be nonabelian. Our notation is standard and may be found in Fuchs $(1970,1973)$ which also contains all the fundamental concepts used herein; an exception is that mappings are written on the right. The monograph of Kaplansky (1969) provides a detailed discussion of the fundamental concepts of transitivity and full transitivity (for $p$-groups).

\section{GENERAL OBSERVATIONS}

In this section we start by introducing the new concept of weak transitivity for abelian groups. 
Definition 1.1 Let $G$ be a group. Then $G$ is called weakly transitive if for any pair of elements $x, y \in G$ there exists an automorphism $\phi$ of $G$ mapping $x$ onto $y$ if and only if there exist endomorphisms $\varphi, \psi$ of $G$ such that $x \varphi=y$ and $y \psi=x$.

Our first aim is to show that it is necessary to understand the torsion-free weakly transitive groups in order to understand the weakly transitive groups in general. Later on we shall deal with the torsion-free case while the torsion case is taken care of in Goldsmith and Strüngmann (submitted).

Recall that a fully invariant (characteristic) subgroup $H$ of a group $G$ is a subgroup that is invariant under all endomorphisms (automorphisms) of $G$.

Lemma 1.2 Let $G$ be a weakly transitive group and $H$ a characteristic summand of $G$, then $H$ is weakly transitive.

Proof. Let $x, y \in H$ and $\varphi, \psi \in \operatorname{End}(H)$ such that $x \varphi=y$ and $y \psi=x$. Since $H$ is a summand of $G$ we can find $\varphi^{\prime}, \psi^{\prime} \in \operatorname{End}(G)$ such that $x \varphi^{\prime}=y$ and $y \psi^{\prime}=x$. Because $G$ is weakly transitive there is an automorphism $\alpha$ of $G$ mapping $x$ onto $y$. By the invariance of $H$ in $G, \alpha \uparrow_{H}$ is an automorphism of $H$. Therefore $H$ is weakly transitive.

The following well-known result is an easy exercise:

Lemma 1.3 Let $G=G_{1} \oplus G_{2}$ be any group such that $\operatorname{Hom}\left(G_{1}, G_{2}\right)=0$. If $\alpha$ is an automorphism of $G$ and $\pi_{1}$ and $\pi_{2}$ are the canonical projections onto $G_{1}$ and $G_{2}$ respectively, then $\left(\alpha \pi_{1}\right) \Gamma_{G_{1}}$ and $\left(\alpha \pi_{2}\right) \Gamma_{G_{2}}$ are automorphisms of $G_{1}$ and $G_{2}$ respectively.

Elementary results on weak transitivity can be summarized in:

Proposition 1.4 The following hold.

(i) Let $G=G_{1} \oplus G_{2}$ be any group such that $\operatorname{Hom}\left(G_{1}, G_{2}\right)=0$. If $G$ is weakly transitive, then $G_{1}$ and $G_{2}$ are weakly transitive. In particular if $G$ is weakly transitive and $G=T \oplus H$ with $T$ torsion and $H$ torsion-free, then $T$ and $H$ are weakly transitive.

(ii) Let $\left\{G_{i}: i \in I\right\}$ be a family of weakly transitive groups with $\operatorname{Hom}\left(G_{i}, G_{j}\right)=0$ for $i \neq j \in I$. Then the direct sum $\bigoplus_{i \in I} G_{i}$ and the cartesian product $\prod_{i \in I} G_{i}$ are weakly transitive.

Proof. Part ( $i$ ) follows easily from Lemma 1.3. To show (ii) it suffices to observe that mappings of a direct sum or cartesian product can be reduced to actions on the components via a combination of canonical injections and projections. 


\section{TORSION-FREE GROUPS}

As we have seen in the last section it is reasonable to study torsion-free weakly transitive groups in order to get insight into the structure of weakly transitive groups.

Recall from the introduction that a torsion-free group $G$ is (fully) transitive if for any $x, y \in G$ with $\left(U_{G}(x) \leq U_{G}(y)\right) U_{G}(x)=U_{G}(y)$ there exists an (endomorphism) automorphism of $G$ mapping $x$ onto $y$. The group $G$ is $P$-local for a set of primes $P$ if $G$ is divisible by all primes not in $P$. Our next result is straight forward but nevertheless is fundamental to our approach.

Lemma 2.5 A torsion-free fully transitive group is transitive if and only if $G$ is weakly transitive.

Lemma 2.6 Let $P$ be a finite set of primes and $G$ be a torsion-free $P$-local group. If $G$ is transitive, then $G$ is fully transitive.

Proof. Let $x, y \in G$ such that $U_{G}(x) \leq U_{G}(y)$. Since $G$ is $P$-local and $P$ is a finite set there exists $y^{\prime} \in G$ such that $U_{G}(x)=U_{G}\left(y^{\prime}\right)$ and $y=n y^{\prime}$ for some natural number $n$. By transitivity there is an automorphism $\alpha$ of $G$ mapping $x$ onto $y^{\prime}$. Hence $n \alpha$ maps $x$ onto $y$ and therefore $G$ is fully transitive.

Corollary 2.7 Let $P$ be a finite set of primes and $G$ be a torsion-free $P$-local group. Then $G$ is transitive if and only if it is fully transitive and weakly transitive.

Proof. Combine Lemma 2.5 and Lemma 2.6.

After having clarified the relationship between (fully) transitive and weakly transitive groups we show that torsion-free weakly transitive groups exist in abundance.

Proposition 2.8 Let $G$ be an abelian group such that any non-trivial endomorphism of $G$ is monic. Then $G$ is weakly transitive.

Proof. Let $0 \neq x, y \in G$ and $\varphi, \psi \in \operatorname{End}(G)$ such that $x \varphi=y$ and $y \psi=x$. Hence $x \varphi \psi=x$ and therefore $x\left(\varphi \psi-i d_{G}\right)=0$. By assumption we obtain $\varphi \psi=i d_{G}$ and similarly $\psi \varphi=i d_{G}$. Thus $\varphi$ (and also $\psi$ ) is an automorphism of $G$ mapping $x$ onto $y$.

Lemma 2.9 Let $G$ be a torsion-free group such that $G$ is cyclic over its endomorphism ring. If $\operatorname{End}(G)$, the endomorphism ring of $G$, is a domain, then $G$ is weakly transitive. 
Proof. Let $R=\operatorname{End}(G)$ and $G=x R$ for some $x \in G$. We claim that every non-trivial endomorphism of $G$ is monic and hence Proposition 2.8 applies. Let $0 \neq \varphi \in R$ and assume that $y \varphi=0$ for some $0 \neq y \in G$. Write $y=x \psi$ with $0 \neq \psi \in R$. Then $x \psi \varphi=0$ but $R$ is commutative, hence $(x \gamma) \psi \varphi=(x \psi \varphi) \gamma=0$ for every $\gamma \in R$. Thus $\psi \varphi=0$ and therefore $\varphi=0$ since $R$ is a domain hence has no zero-divisors - contradiction.

For the following examples recall that a commutative unital ring is an E-ring if all endomorphisms of the additive group of $R$ are multiplications by elements of $R$.

Example 2.10 Let $G$ be a torsion-free group. Then $G$ is weakly transitive if any of the following conditions is satisfied:

(i) $G$ is of finite rank and $\operatorname{End}(G)$ has no non-trivial zero-divisors;

(ii) $G$ is of finite rank and $\mathbb{Q} \otimes \operatorname{End}(G)$ is a division algebra;

(iii) $G$ is the additive group of an $E$-ring $R$ which is a domain.

Proof. By Arnold (1980) Proposition 6.1 the assumptions in (i) and (ii) are equivalent to the property that every non-trivial endomorphism of $G$ is monic. Hence Proposition 2.8 implies that $G$ is weakly transitive. To prove (iii) note that $G$ is trivially cyclic over its endomorphism ring and hence Lemma 2.9 gives the desired result.

Note that $E$-rings which are domains exist in abundance; the construction of an $E$-ring of arbitrary large cardinality given in Dugas, Mader and Vinsonhaler (1987) will yield a domain provided the initial ring used is a domain.

For the last theorem of this section assume that $R$ is an arbitrary ring with 1 and let $S$ be a countable multiplicatively closed subset of $Z(R)$, the center of $R$ consisting of non zero-divisors. Hence $S$ induces a Hausdorff topology on any $S$-reduced and $S$-torsion-free $R$-module $M$ by choosing $M q_{n}$ as a basis of neighbourhoods of 0 where $q_{n}=s_{1} \cdots s_{n}$ for $n \in \omega$. Here we say that $M$ is $S$-torsion-free if $m s \neq 0$ for $0 \neq m \in M$ and $s \in S$; and $M$ is $S$-reduced if $\bigcap_{s \in S} M s=0$. Finally, $M$ is $S$-cotorsion-free if $\operatorname{Hom}(\hat{R}, M)=0$, where $\hat{R}$ is the completion of $R$ in the topology induced by $S$ (see also Corner and Göbel (1985)). We shall assume that $R$ is $S$-reduced and $S$-torsion-free. Note that if $R$ has no zero-divisors, then $S=\mathbb{Z}$ or $S=\left\{p^{n}: n \in \omega\right\}$ ( $p$ a prime) could be chosen.

Theorem 2.11 Let $R$ have no zero-divisors. If $R$ is $S$-reduced, $S$-torsion-free and $S$ cotorsion-free, then $R$ is the endomorphism ring of a weakly transitive torsion-free group of arbitrarily large cardinality. 
Proof. It is well-known (see Corner and Göbel (1985)) that such a ring $R$ can be realized as the endomorphism ring of a torsion-free abelian group $G$ of arbitrarily large cardinality. We claim that all endomorphisms of $G$ are monic, hence Proposition 2.8 implies that $G$ is weakly transitive. Let $\lambda=|G|$. By the standard construction $G$ is the union of a smooth increasing sequence of subgroups $G_{\alpha}(\alpha<\lambda)$. Moreover, $G$ is sandwiched between a free

$R$-module $G_{0}=B=\bigoplus_{\alpha<\lambda} e_{\alpha} R$ and its completion $\hat{B}$ in the $S$-topology on $B$. Since $B$ is $R$-torsion-free also its completion (and therefore $G$ ) is $R$-torsion-free. Thus each endomorphism of $R$ is monic and hence $G$ is weakly transitive.

We would like to remark that one can weaken the assumption in the above theorem to the property that $R$ is right-cancelable which means that $r r^{\prime}=0$ for $r, r^{\prime} \in R$ implies $r^{\prime}=0$. Note that this shows the existence of weakly transitive groups with highly pathological decompositions; by realizing an appropriate ring of Corner-Leavitt type - see e.g. Fuchs (1973) Theorem 91.6 - there exists a weakly transitive torsion-free abelian group $G$ such that $G \cong G^{3}$ but $G \nsubseteq G^{2}$. Hence $G$ and $G^{3}$ are weakly transitive and it would be interesting to know if also $G^{2}$ is weakly transitive (see Theorem 3.33 ).

\section{SEPARABLE TORSION-FREE GROUPS}

In this section we shall consider separable torsion-free groups, in particular completely decomposable groups. Recall that a completely decomposable group is a direct sum of subgroups of the rationals $\mathbb{Q}$. In the sequel we shall use notations as in Mader (2000). A rational group $R$ (a subgroup of $\mathbb{Q}$ ) is uniquely determined by its type which will usually be denoted by $t p(R)$. The set of types form a lattice and a type $\tau$ is called idempotent if $\tau=t p(R)$ for some subring $R$ of $\mathbb{Q}$. Since there is no loss of generality we will always assume that a rational group $R$ contains 1 . If $G$ is a torsion-free group, then the type $t p(x)$ of an element $x \in G$ is $t p\left(\langle x\rangle_{*}\right)$, the type of the pure subgroup generated by $x$ inside $G$. The height sequence or characteristic $\left(h t_{p_{1}}(x), h t_{p_{2}}(x), \cdots\right)$ of $x$ is denoted by $\chi(x)$. Finally, the typeset $T s t(G)$ of a torsion-free group $G$ is the set $\{t p(x): x \in G\}$ and if $\tau \in T s t(G)$, then the type subgroups are defined by

$$
\begin{aligned}
G(\tau) & =\{x \in G: \operatorname{tp}(x) \geq \tau\} \\
G^{\sharp}(\tau) & =\langle x \in G: \operatorname{tp}(x)>\tau\rangle_{*} .
\end{aligned}
$$

The critical typeset $T_{c r}(G)$ is the set of all types $\tau \in T \operatorname{st}(G)$ such that $G(\tau) / G^{\sharp}(\tau)$ is nontrivial. For further results and details on completely decomposable groups we refer to Mader (2000). 
The first two examples show that a completely decomposable group of finite rank need be neither transitive nor fully transitive.

Example 3.12 Let $R$ and $S$ be two rational groups of incomparable idempotent type and let $p$ be a prime such that $R$ is $p$-divisible but $S$ is not. Then $S \oplus R$ is not (fully) transitive.

Proof. Since $R$ and $S$ are of incomparable type it is clear that any (endomorphism) automorphism of $S \oplus R$ is of the form $\alpha \oplus \beta$ where $\alpha$ is an (endomorphism) automorphism of $R$ and $\beta$ an (endomorphism) automorphism of $S$. Obviously, the elements $(1,1)$ and $(p, 1)$ have the same characteristic in $S \oplus R$ but can not be mapped onto each other by an (endomorphism) automorphism of $S \oplus R$.

Even a completely decomposable group with linearly ordered critical typeset need not be (fully) transitive.

Example 3.13 Let $\tau_{1}=\mathbb{Z}, \tau_{2}=\mathbb{Z}^{(2)}$, and let $A=\tau_{1} v_{1} \oplus \tau_{2} v_{2}$. Then $T_{c r}(A)=\left\{\tau_{1}, \tau_{2}\right\}$ is linearly ordered but $A$ is not (fully) transitive.

Proof. Consider the element $x=3 v_{1}+v_{2}$. Then $t p(x)=\mathbb{Z}=t p\left(v_{1}\right)$. In particular, $\mathbb{Z} x$ is pure in $A$, but it cannot be a direct summand as $A=\mathbb{Z} x \oplus B$ would imply that $B=A^{\sharp}\left(\tau_{1}\right)=\tau_{2} v_{2}$ and further that $A=\tau_{1}\left(3 v_{1}\right) \oplus \tau_{2} v_{2}$. There cannot be an (endomorphism) automorphism $\alpha$ of $A$ with $x \alpha=v_{1}$ since this would imply that $A=\mathbb{Z} x \oplus \tau_{2} v_{2}$ $\left(\frac{1}{3} \notin \operatorname{End}(\mathbb{Z})\right)$.

Let us remark that generalizing the above examples one can show that the only (fully) transitive completely decomposable groups of finite rank are in fact the homogeneous ones.

Lemma 3.14 A homogeneous completely decomposable group $H$ is weakly transitive, transitive and fully transitive.

Proof. It suffices to prove that $H$ is fully transitive and transitive since transitivity implies weak transitivity. Let $x, y \in H$ such that $\chi(x)=\chi(y)(\chi(x) \leq \chi(y))$ By Fuchs (1973) Lemma 86.8 we obtain $H=\langle x\rangle_{*} \oplus H_{1}$ and $H=\langle y\rangle_{*} \oplus H_{2}$. Clearly, $H_{1}$ and $H_{2}$ are isomorphic, say $\alpha$ is an isomorphism. It is easy to see that we can construct an isomorphism (endomorphism) $\beta:\langle x\rangle_{*} \rightarrow\langle y\rangle_{*}$ which maps $x$ onto $y$. The sum $\alpha \oplus \beta$ is as required.

Corollary 3.15 If $\left\{C_{\tau}: \tau \in T\right\}$ is a family of completely decomposable groups such that each $C_{\tau}$ is $\tau$-homogeneous and $T$ is an antichain, then $\bigoplus_{\tau \in T} C_{\tau}$ and $\prod_{\tau \in T} C_{\tau}$ are weakly transitive. 
Proof. Follows by Proposition 1.4.

We now intend to characterize the weakly transitive completely decomposable groups. Therefore we need the following

Lemma 3.16 Let $C$ be a completely decomposable group and let $R$ be a rational group such that $\tau \leq t p(R)$ for all $\tau \in T_{c r}(C)$. If $x \in C$ and $y \in R$ are such that $\chi_{C}(x) \leq \chi_{R}(y)$, then there is a mapping $\varphi \in \operatorname{Hom}(C, R)$ such that $x \varphi=y$.

Proof. Since any element $x \in C$ is contained in a finite rank summand of $C$ we may assume without loss of generality that $C$ is of finite rank. We induct on the rank $r k(C)=n$ of $C$. If $n=1$, then the claim is easily established. Therefore let $n>1$ and write $C=C^{\prime} \oplus S$ with $S \subseteq \mathbb{Q}$. Without loss of generality $x=c^{\prime}+s$ with $c^{\prime} \in C^{\prime}$ and $0 \neq s \in S$. Now $h t_{p}(x)=\min \left\{h t_{p}\left(c^{\prime}\right), h t_{p}(s)\right\}$ for all primes $p$ and by hypothethis $t p\left(c^{\prime}\right) \leq t p(y)$ and $t p(s) \leq t p(y)$. Let $m$ and $k$ be minimal with $\chi\left(c^{\prime}\right) \leq \chi(m y)$ and $\chi(s) \leq \chi(k y)$. We claim that $\operatorname{gcd}(m, k)=1$. If $p \mid m$ and $p \mid k$, then $h t_{p}\left(c^{\prime}\right)>h t_{p}(y)$ and $h t_{p}(s)>h t_{p}(y)$, hence $h t_{p}(x)>h t_{p}(y)$, a contradiction. Therefore $\operatorname{gcd}(m, k)=1$ and there are $l_{1}, l_{2}$ such that $1=l_{1} m+l_{2} k$, thus $y=l_{1} m y+l_{2} k y$. By induction we can map $c^{\prime}$ onto $l_{1} m y$ with $\psi_{1} \in \operatorname{Hom}\left(C^{\prime}, R\right)$ and $s$ onto $l_{2} k y$ with $\psi_{2} \in \operatorname{Hom}(S, R)$. So $\psi_{1} \oplus \psi_{2} \in \operatorname{Hom}(C, R)$ is as required.

For the next Theorem we need some further notation. Let $R$ be a rational group. By $E(R)=\left\{p \in \Pi \mid \frac{1}{p} \in \operatorname{End}(R)\right\}=\{p \in \Pi \mid R$ is $p-$ divisible $\}$ we denote the set of all primes $p$ such that multiplication by $p$ is an automorphism of $R$. Moreover, we let $U(R)=\langle E(R)\rangle$ be the multiplicative group generated by $E(R)$, i.e. any element $r \in U(R)$ is of the form $r=\prod_{i \in I} p_{i}^{n_{i}}$ with $I$ a finite index set and $p_{i} \in E(R), n_{i} \in \mathbb{Z}$. Note that $U(R)$ is isomorphic to the automorphism group of $R$.

Theorem 3.17 Let $C$ be a completely decomposable group of finite rank. If $R$ is a rational group of type strictly greater than all types in $T_{c r}(C)$, then $R \oplus C$ is weakly transitive if and only if

(i) $C$ is weakly transitive;

(ii) for any pair of integers $m, n$ such that $\operatorname{gcd}(m, n)=1$ and $\operatorname{gcd}(m, p)=\operatorname{gcd}(n, p)=1$ for all $p \in E(R)$ there is an element $u \in U(R)$ and a rational number $r \in R$ such that $u m+r n=1$.

Condition (ii) is equivalent to 1 in the stable range of $R$. 
Proof. Assume that (i) and (ii) hold. Let $x=\left(x_{R}, x\right), y=\left(y_{R}, y\right) \in R \oplus C$ and $\varphi, \psi \in$ $\operatorname{End}(R \oplus C)$ such that $\left(x_{R}, x\right) \varphi=\left(y_{R}, y\right)$ and $\left(y_{R}, y\right) \psi=\left(x_{R}, x\right)$. Since $\operatorname{Hom}(R, C)=0$ it follows that there are endomorphisms of $C$ mapping $x$ onto $y$ and conversely. By $(i)$ there is an automorphism $\gamma$ of $C$ mapping $x$ onto $y$. Moreover, we have $y_{R}=x_{R} \alpha+x \beta$ with $\alpha \in \operatorname{End}(R)$ and $\beta \in \operatorname{Hom}(C, R)$. Hence $\alpha=\frac{m}{n}$ with $\frac{1}{n}$ an automorphism of $R$ and $\operatorname{gcd}(m, p)=1$ for all $p \in E(R)$. Clearly it suffices to assume that $n=1$. So $y_{R}=m x_{R}+x \beta$. By assumption there is a minimal integer $k$ such that $\chi\left(k x_{R}\right) \geq \chi(x)$. Obviously $\operatorname{gcd}(k, p)=1$ for all $p \in E(R)$ can be chosen and we claim that also $\operatorname{gcd}(m, k)=1$. If $p \mid k$, then $h t_{p}\left(x_{R}\right)<h t_{p}(x)$, hence $h t_{p}\left(x_{R}\right)=h t_{p}\left(y_{R}\right)$. If also $p \mid m$, then $h t_{p}\left(y_{R}\right) \geq \min \left\{h t_{p}\left(m x_{R}\right), h t_{p}(x \beta)\right\} \geq h t_{p}\left(x_{R}\right)+1-$ a contradiction. Thus $\operatorname{gcd}(m, k)=1$.

By $(i i)$ there is $u \in U(R)$ and $r \in R$ such that $1=u m+k r$. Hence $u y_{R}=u m x_{R}+u x \beta=$ $x_{R}-\left(k r x_{R}-u x \beta\right)$. Now $\chi\left(k r x_{R}-u x \beta\right) \geq \chi(x)$, hence Lemma 3.16 implies that there is a map $\beta: C \rightarrow R$ such that $x \beta=k r x_{R}-u x \beta$. We define $\delta \in \operatorname{Aut}(R \oplus C)$ by $(s, c) \delta=(s-c \beta, c \gamma)$ and obtain $\left(x_{R}, x\right) \delta=\left(u y_{R}, y\right)$. Since $u$ is an automorphism of $R$ there is an automorphism of $R \oplus C$ mapping $\left(u y_{R}, y\right)$ onto $\left(y_{R}, y\right)$ and we are finished.

Conversely, assume that $R \oplus C$ is weakly transitive. Then Lemma 1.4 implies that $C$ is weakly transitive as well. It remains to prove $(i i)$. Let $m, n$ be given such that $\operatorname{gcd}(m, n)=$ $1=\operatorname{gcd}(m, p)=\operatorname{gcd}(n, p)$ for all $p \in E(R)$. Hence there are integers $l, k$ such that $k m+l n=1$. Choose any summand $S$ of $C$ of rank 1 , say $C=S \oplus C^{\prime}$. Then the mapping $\varphi \in \operatorname{End}\left(R \oplus S \oplus C^{\prime}\right)$ defined by $(r, s, c) \varphi=(k r+l s, s, c)$ maps $(m, n, 0)$ onto $(1, n, 0)$ and conversely the mapping $\psi$ defined by $(r, s, c) \psi=(m r, s, c)$ maps $(1, n, 0)$ onto $(m, n, 0)$. By weak transitivity there is an automorphism $\alpha$ of $R \oplus S \oplus C^{\prime}$ mapping $(m, n, 0)$ onto $(1, n, 0)$. Thus $1=u m+r n$ for some $u \in \operatorname{End}(R)$ and $r \in \operatorname{Hom}(S, R)$. Since $\operatorname{Hom}(R, C)=\operatorname{Hom}(R, S)=0$ it follows that $u$ is an automorphism of $R$ by Lemma 1.3, hence $u \in U(R)$. Clearly, $r \in R$ and this finishes the equivalence of $(i)$ and $(i i)$. For rational rings $R$ condition $(i i)$ of Theorem 3.17 is clearly equivalent to 1 being in the stable range of $R$. If $R$ is a rational group, then it has been observed by Meehan and Strüngmann (submitted) that $R$ satisfies condition (ii) of Theorem 3.17 if and only if 1 is in the stable range of $\operatorname{End}(R)$. This finishes the proof.

For the next result recall that a set of primes $P$ is cofinite if its complement $\Pi \backslash P$ is finite.

Lemma 3.18 Let $R \cong \mathbb{Z}^{(P)}$ for some cofinite set of primes $P$. Then condition (ii) of Theorem 3.17 holds.

Proof. Let $\Pi \backslash P=\left\{p_{1}, \cdots, p_{t}\right\}$ and suppose that $m, n$ are integers with $\operatorname{gcd}(m, n)=1$ and $\operatorname{gcd}(m, p)=\operatorname{gcd}(n, p)=1$ for all $p \in P$. Put $J=\{p \in \Pi \backslash P: p \wedge n, p \wedge m\}$ and $n^{\prime}=n \prod_{p \in J} p$. We will show that there exist integers $a, b$ such that $a m-b n^{\prime}=1$ and $\operatorname{gcd}(a, m)=1$. Since obviously $\operatorname{gcd}\left(a, n^{\prime}\right)=1$ we obtain that $\operatorname{gcd}(a, p)=1$ for all $p \in P$ which implies that $a$ is a unit in $R$, hence $a \in U(R)$ and therefore condition (ii) of Theorem 
3.17 holds.

Since $\left(m, n^{\prime}\right)=1$ there exist integers $l_{1}, l_{2}$ with $1=l_{1} m-l_{2} n^{\prime}$. If $\operatorname{gcd}\left(l_{1}, m\right)=1$ we are finished; suppose $m=p^{r_{1}} \cdots p_{k}^{r_{k}} p_{k+1}^{r_{k+1}} \cdots p_{t}^{r_{t}}$ where $p_{1}, \cdots, p_{k} \mid l_{1}$, but $p_{k+1}, \cdots, p_{t} \chi l_{1}$. Then $1=\left(l_{1}+p_{k+1} \cdots p_{t} n^{\prime}\right) m-\left(l_{2}+p_{k+1} \cdots p_{t} m\right) n^{\prime}=a m-b n^{\prime}$. We claim that $\operatorname{gcd}(a, m)=1$. If some $p_{i}(1 \leq i \leq k)$ divides $a$, then since $p_{i} \mid l_{1}$ we have $p_{i} \mid p_{k+1} \cdots p_{t} n^{\prime}$, hence $p_{i} \mid n$ - a contradiction. So no $p_{i}(1 \leq i \leq k)$ divides $a$. On the other hand, if some $p_{j}(k+1 \leq j \leq t)$ divides $a$ then $p_{j} \mid l_{1}$ - again a contradiction. So no $p_{j}(k+1 \leq j \leq t)$ divides $a$. Thus $\operatorname{gcd}(a, m)=1$.

Recall that a torsion-free group $G$ is separable if every finite set of elements of $G$ is contained in a finite rank completely decomposable summand of $G$.

Lemma 3.19 If $G$ is a separable group, then $G$ is weakly transitive if every finite rank summand of $G$ is weakly transitive.

Proof. Let $x, y \in G$ and $x \varphi=y, y \psi=x$ for some endomorphisms $\varphi, \psi \in \operatorname{End}(G)$. Since $G$ is separable there is a direct summand $H$ of $G$ which is completely decomposable of finite rank and contains $x$ and $y$. Let $\pi$ be the projection onto $H$ along a fixed complement of $H$ in $G$. Obviously, $(\varphi \pi) \uparrow_{H}$ and $(\psi \pi) \uparrow_{H}$ are endomorphisms of $H$ mapping $x$ onto $y$ and conversely. Since every automorphism of $H$ extends to an automorphism of $G$ and $H$ is weakly transitive it follows that $G$ is weakly transitive.

For the next theorem recall that a $P$-local group for some set of primes $P$ is a group which is divisible by all primes $p \notin P$.

Theorem 3.20 Let $C$ be a P-local separable group for some finite set of primes $P$. Then $C$ is weakly transitive.

Proof. Let $x, y \in C$ and $x \varphi=y, y \psi=x$ for some endomorphisms $\varphi, \psi \in \operatorname{End}(C)$. Without loss of generality we may assume that $C$ is of finite rank and completely decomposable by Lemma 3.19. We induct on the rank $n=r k(C)$ of $C$. If $n=1$, then $C$ is homogeneous and therefore weakly transitive by Lemma 3.14. Assume $n>1$ and let $R$ be an arbitrary maximal type in $T_{c r}(C)$ and let $C=C_{R} \oplus C^{\prime}$ with $C_{R}$ a maximal $t p(R)$-homogeneous completely decomposable summand of $C$. Let $x=x_{R}+x^{\prime}$ and $y=y_{R}+y^{\prime} \in C_{R} \oplus C^{\prime}$. Since $\operatorname{Hom}\left(C_{R}, C^{\prime}\right)=0$ it follows that there are endomorphisms of $C^{\prime}$ mapping $x^{\prime}$ onto $y^{\prime}$ and conversely. By induction there is $\alpha \in A u t\left(C^{\prime}\right)$ such that $x^{\prime} \alpha=y^{\prime}$ and so the automorphism $\alpha \oplus i d_{C^{\prime}}$ of $C$ maps $x_{R}+x^{\prime}$ onto $x_{R}+y^{\prime}$. We now distinguish two cases:

a) If there are two maximal types $R_{1}, R_{2}$ in $T_{c r}(C)$, then write $x=x_{R_{1}}+x_{R_{2}}+x^{\prime \prime}$ and $y=y_{R_{1}}+y_{R_{2}}+y^{\prime}$ in a fixed decomposition $C=C_{R_{1}} \oplus C_{R_{2}} \oplus C^{\prime}$ of $C$ where $C_{R_{i}}$ are maximal $t p\left(R_{i}\right)$-homogeneous completely decomposable summands of $C$ for $i=1,2$. By the above there is an automorphism $\alpha$ of $C$ mapping $x_{R_{2}}+x_{R_{1}}+x^{\prime \prime}$ onto $x_{R_{2}}+y_{R_{1}}+y^{\prime}$. Again by 
the same argument there is an automorphism $\beta$ of $C^{\prime}$ mapping $x_{R_{2}}+y^{\prime}$ onto $y_{R_{2}}+y^{\prime}$ which extends to an automorphism of $C$ mapping $x$ onto $y$.

b) It remains to consider the case when there is just one maximal type in $T_{c r}(C)$. By induction $C^{\prime}$ is weakly transitive and by Lemma 3.18 condition (ii) of Theorem 3.17 holds. Applying Theorem 3.17 shows that $C$ is weakly transitive.

Next we want to find completely decomposable groups which are not weakly transitive. To do so we have to find rational groups violating condition (ii) of Theorem 3.17.

Lemma 3.21 Let $R \subseteq \mathbb{Q}$ be such that condition (ii) of Theorem 3.17 holds. Then for any integer $m$ there is $r \in R$ such that $r m$ is the sum of at most two units in $R$. In particular, if $R$ is a ring, then $r$ can be chosen to be an integer.

Proof. Let $m$ be any integer. If $m \in U(R)$ we are finished. Hence assume $m \notin U(R)$. Since $(m, 1)=1$ there are $r \in R$ and $u \in U(R)$ sucht that $r m+u=1$. Thus $r m=1-u$ is the sum of two units. If $R$ is a ring, then $r=\frac{n}{k}$ with $\frac{1}{k} \in U(R)$, hence $n m=k-k u$ is a sum of two units.

Corollary 3.22 If $R \subseteq \mathbb{Q}$ and $\operatorname{End}(R) \cong \mathbb{Z}$, then $R$ does not satisfy condition (ii) of Theorem 3.17.

Proof. Follows easily from Lemma 3.21. Alternatively, by Arnold (1980) Example 8.3. 1 is not in the stable range of $\mathbb{Z}$. Thus $R$ can not satisfy condition (ii) of Theorem 3.17 .

Unfortunately, rational groups $R$ which are divisible by at least one prime may have the property that a multiple of any integer is the sum of at most two units in $R$. Therefore, different techniques are required in order to violate condition (ii) of Theorem 3.17 for those groups.

Lemma 3.23 Let $m$ be a rational and $p_{1}, \cdots, p_{n}$ odd primes. If $N=\prod_{i \leq n} p_{i}>2^{m} 2^{m}$, then $\prod_{i \leq n}\left(p_{i}-1\right)>2^{m}$.

Proof. We induct on $n$. If $n=1$, then $N=p$, so $p-1>2^{m}$. If $n>1$ we let $n=n^{\prime}+1$, and choose an integer $k$ maximal subject to $2^{k}<p_{n}$; then $k \geq 1$ and $2^{k}+1 \leq p_{n}<2^{k+1}$. Hence $\prod_{i \leq n^{\prime}} p_{i}>2^{m-k} 2^{m-k}$ because if $\prod_{i \leq n^{\prime}} p_{i} \leq 2^{m-k} 2^{m-k}$, then $\prod_{i \leq n} p_{i} \leq 2^{m-k} 2^{m-k} p_{n}<$ $2^{m-k} 2^{m-k+k+1}<2^{m-k+1} 2^{m}<2^{m} 2^{m}-$ a contradiction. Thus by induction $\prod_{i \leq n^{\prime}}\left(p_{i}-1\right)>$ $2^{m-k}$ and so $\prod_{i \leq n}\left(p_{i}-1\right)>2^{m}$. 
Lemma 3.24 If $R \subseteq \mathbb{Q}$ and $\operatorname{End}(R) \cong \mathbb{Z}^{(p)}$ for some prime $p$, then condition (ii) of Theorem 3.17 does not hold.

Proof. Let $R \subseteq \mathbb{Q}$ and assume that $\operatorname{End}(R) \cong \mathbb{Z}^{(p)}$. Choose an integer $m$ such that $2^{m}>$ $4(2 m+1)$, hence $-1+p^{2 m+1}>2^{2 m}=2^{m} 2^{m}>4(2 m+1)$. Write $\left(-1+p^{2 m+1}\right)=\prod_{i \leq n} p_{i} 2^{l}$ with $n, l \in \mathbb{N}$ and $p_{i}$ odd primes, not necessarily distinct. Thus $\prod_{i \leq n} p_{i}>2^{2 m-l}$ and hence $\prod_{i \leq n}\left(p_{i}-1\right)>2^{\left(m-\frac{l}{2}\right)}$ by Lemma 3.23. Put $a=\left(-1+p^{2 m+1}\right)$ and let $G_{a}$ be the multiplicative group of all integers less than $a$ which are relatively prime to $a$. The multiplication in $G_{a}$ is given by ordinary multiplication modulo $a$. It is well-known that $G_{a}$ has cardinality $\left|G_{a}\right| \geq 2^{l-1} \prod_{i \leq n}\left(p_{i}-1\right)$ and hence $\left|G_{a}\right|>2^{l-1} 2^{\left(m-\frac{l}{2}\right)} \geq 2^{m-1}>2(2 m+1)$. Let $H \subseteq G_{a}$ be the subgroup of $G_{a}$ generated by $p$. Note that $\operatorname{gcd}(a, p)=1$. Since $p^{(2 m+1)} \equiv+1$ modulo $a$ the size of $H$ is at most $2 m+1$, hence $\left|G_{a}\right|>2|H|$. Thus the quotient group $G_{a} / H$ has at least three cosets and therefore there is $t<a$ with $\operatorname{gcd}(t, a)=1$ and $t H$ does not contain 1 or -1 . Hence $t p^{s} \not \equiv 1$ for every $s \in \mathbb{N}$. Put $b=a+t$, hence $\operatorname{gcd}(b, a)=1$. Write $b=p^{i} b^{\prime}$ with $i \in \mathbb{N}$ and $\operatorname{gcd}\left(b^{\prime}, p\right)=1$. Moreover, let $v=\prod_{q \mid a} q^{h t_{q}^{R}(1)}$ and put $a^{\prime}=v a$. Then we still have that $\operatorname{gcd}\left(b^{\prime}, a^{\prime}\right)=\operatorname{gcd}\left(b^{\prime}, p\right)=\operatorname{gcd}\left(a^{\prime}, p\right)=1$. Assume that condition (ii) of Theorem 3.17 holds. Then there is a unit $u \in U(R)$ and an element $r \in R$ such that $u b^{\prime}+r a^{\prime}=1$, thus $u b^{\prime}+r v a=u p^{-i} a+u p^{-i} t+r v a=1$. An easy calculation shows that $r v \in \mathbb{Z}$ by the choice of $v$, hence $u a+u t+p^{i} r v a=p^{i}$ and so $u t \equiv p^{i}$ modulo $a$. Let $u=p^{z}$, then $p^{(z-i)} t \equiv 1$ modulo $a$ - a contradiction. Therefore condition (ii) of Theorem 3.17 does not hold.

Obviously, if $R$ is a rational group and $\operatorname{End}(R)$ satisfies condition (ii) from Theorem 3.17, then also $R$ satisfies it. As we have seen condition (ii) holds if $R$ contains only finitely many primes which are not units (Lemma 3.18). On the other hand, Corollary 3.22 and Lemma 3.24 show that it is plausible to conjecture that $R$ does not satisfy condition (ii) of Theorem 3.17 if there are only finitely many primes which are units in $R$. However, we are unable to establish this. The next examples show that in the case of an infinite and coinfinite set of primes being units both situations can occur. ${ }^{1}$

Example 3.25 Let $P=\{p \in \Pi: p \equiv 11$ modulo 120$\}$. Then $R=\mathbb{Z}^{(P)}$ does not satisfy condition (ii) of Theorem 3.17.

Proof. Choose $a=91$ and $b=60$, then $\operatorname{gcd}(a, b)=1$. Hence $a \equiv 91$ modulo 120 and $r b \equiv 60$ or $r b \equiv 0$ modulo 120 for every $r \in R$. Easy calculations show that a unit $u \in R$ must satisfy $u \equiv s$ modulo 120 for some $s \in\{-11,-1,1,11\}$. Hence $a+r b \not \equiv u$ modulo

\footnotetext{
${ }^{1}$ We would like to thank Chris Meehan for suggesting the examples 3.25, 3.26 and 3.27.
} 
120 for every $r \in R$ and any unit $u \in R$. Thus condition (ii) of Theorem 3.17 does not hold.

In the above example we could also choose $P=\{p \in \Pi: p \equiv 13$ modulo 168$\}, P=\{p \in$ $\Pi: p \equiv 7$ modulo 48$\}$, or $P=\{p \in \Pi: p \equiv 17$ modulo 288 $\}$. However, not all congruence classes work.

Example 3.26 Let $P=\{p \in \Pi: p \not \equiv 1$ modulo 24$\}$. Then $R=\mathbb{Z}^{(P)}$ satisfies condition (ii) of Theorem 3.17.

Proof. Let $a, b \in \mathbb{Z}$ with $\operatorname{gcd}(a, b)=1$ and $\operatorname{gcd}(a, p)=\operatorname{gcd}(b, p)=1$ for all $p \in P$. Without loss of generality we may assume that $a>b$ and that $a, b$ are positive integers. By Dirichlet's Theorem Dedekind and Dirichlet (1968) Page 359 the set $\{2 a+k(3 b): k \in \mathbb{N}\}$ contains an infinite number of primes. Modulo 24 the possible congruences of these primes are $\{2 a+3 b, 2 a+9 b, 2 a+15 b, 2 a+21 b\}$. Using that $p \equiv 1$ for all prime divisors $p$ of either $a$ or $b$ this set becomes $\{5,11,17,23\}$. Thus if $2 a+k(3 b)=p \in \Pi$, then $p \not \equiv 1$ modulo 24 and therefore $p$ is a unit of $R$. Hence condition (ii) of Theorem 3.17 holds.

For the last example recall that $\pi(n)=|\{p \in \Pi: p<n\}|$ for any $n \in \mathbb{N}$.

Example 3.27 Let $R$ be a subring of $\mathbb{Q}$ and assume that for every integer $n$ there exists some $q_{n} \in \Pi$ with $q_{n}>n$ such that all $p \in \Pi$ with $q_{n} \leq p<q_{n}^{\pi\left(q_{n}\right)+1}$ are elements of $E(R)$. Then condition (ii) of Theorem 3.17 holds.

Proof. Let $0 \neq a, b \in \mathbb{Z}$ such that $\operatorname{gcd}(a, b)=1$. Choose any $q_{n} \in \Pi$ with $n \geq \max (|a|,|b|)$. Put $u=a+\left(\prod_{p \in P} p\right) b$ where $P=\left\{p \in \Pi: p \notin E(R), p<q_{n}, p \vee a, p \vee b\right\}$. We claim that $u<q_{n}^{\pi\left(q_{n}\right)+1}$ and hence $u \in U(R)$. Clearly, $\left(\prod_{p \in P} p\right) b<q_{n}^{|P|+1}$ and therefore

$$
u=a+\left(\prod_{p \in P} p\right) b<q_{n}^{|P|+1}+q_{n}<q_{n}^{|P|+2} \leq q_{n}^{\pi\left(q_{n}\right)+1}
$$

It seems that characterizing those rational groups which satisfy condition (ii) of Theorem 3.17 is a difficult number theoretical problem which we were unable to solve. Therefore we pose the following

Question 3.28 Which $R \subseteq \mathbb{Q}$ satisfy condition (ii) of Theorem 3.17?

Theorem 3.29 Let $C$ be a completely decomposable group. If there exists a rational group $R$ such that $\tau=\operatorname{tp}(R) \in \operatorname{Tcr}(C)$ and 
(i) $\operatorname{rk}\left(C(\tau) / C^{\sharp}(\tau)=1\right.$;

(ii) $\tau$ is not minimal in $T_{c r}(C)$;

(iii) $\tau(p)=\infty$ for at most one prime $p$,

then $C$ is not weakly transitive.

Proof. Assume that $C$ is weakly transitive. Let $C=\bigoplus_{i \in I} C_{i}$ with $C_{i} \subseteq \mathbb{Q}$. Let $I^{\prime}=\left\{i \in I: t p\left(C_{i}\right) \leq \tau\right\}$, then $C=\bigoplus_{i \in I^{\prime}} C_{i} \oplus \underset{i \in I \backslash I^{\prime}}{\bigoplus} C_{i}$ and $\operatorname{Hom}\left(\underset{i \in I \backslash I^{\prime}}{\bigoplus} C_{i}, \bigoplus_{i \in I} C_{i}\right)=0$. Hence Proposition 1.4 implies that $\bigoplus_{i \in I^{\prime}} C_{i}=R \oplus \tilde{C}$ is weakly transitive. By Corollary 3.22 and Lemma 3.24 condition (ii) of Theorem 3.17 does not hold and therefore $R \oplus \tilde{C}$ is not weakly transitive, a contradiction.

In contrast to the above results we obtain

Theorem 3.30 Let $C$ be a completely decomposable, weakly transitive group of finite rank. If $R \subseteq \mathbb{Q}$ and $t p(R)>\tau$ for all $\tau \in T_{c r}(C)$, then $C \oplus R^{n}$ is weakly transitive for all $n>1$.

Proof. Let $x, y \in C \oplus R^{n}$ with $n>1$ and $x \varphi=y, y \psi=x$ for some endomorphisms $\varphi, \psi \in \operatorname{End}\left(C \oplus R^{n}\right)$. Let $C=\bigoplus_{i \leq k} C_{i}$ for some $k \in \mathbb{N}$ and rational groups $C_{i} \subseteq \mathbb{Q}$. Write $x=\left(c_{1}, \cdots, c_{k}, r_{1}, \cdots, r_{n}\right)$ with $c_{i} \in C_{i}$ and $r_{j} \in R$ for $1 \leq i \leq k, 1 \leq j \leq n$. Similarly let $y=\left(c_{1}^{\prime}, \cdots, c_{k}^{\prime}, r_{1}^{\prime}, \cdots, r_{n}^{\prime}\right)$. Since the groups $C_{i}$ and $R$ are rational groups we can write $c_{i}=\frac{\bar{c}_{i}}{\bar{c}_{i}}, c_{i}^{\prime}=\frac{\bar{c}_{i}^{\prime}}{\hat{c}_{i}^{\prime}}$ and $r_{i}=\frac{\bar{r}_{i}}{\hat{r}_{i}}, r_{i}^{\prime}=\frac{\bar{r}_{i}^{\prime}}{\hat{r}_{i}^{\prime}}$ with $\left(\bar{c}_{i}, \hat{c}_{i}\right)=\left(\bar{c}_{i}^{\prime}, \hat{c}_{i}^{\prime}\right)=\left(\bar{r}_{i}, \hat{r}_{i}\right)=\left(\bar{r}_{i}^{\prime}, \hat{r}_{i}^{\prime}\right)=1$. Let $t=\operatorname{lcm}\left(\hat{c}_{i}, \hat{c}_{i}^{\prime}, \hat{r}_{j}, \hat{r}_{j}^{\prime}: 1 \leq i \leq k, 1 \leq j \leq n\right)$. If there exists an automorphism $\alpha$ of $C \oplus R^{n}$ such that $t x \alpha=t y$, then $x \alpha=y$, hence we may assume without loss of generality that all entries $c_{i}, c_{i}^{\prime}, r_{j}, r_{j}^{\prime}$ are integers. Moreover, writing $c_{i}=u_{i} \bar{c}_{i}, c_{i}^{\prime}=u_{i}^{\prime} \bar{c}_{i}^{\prime}, r_{j}=\hat{u}_{j} \bar{r}_{j}, r_{j}^{\prime}=\hat{u}_{j} \bar{r}_{j}^{\prime}$ with $\left(\bar{c}_{i}, p\right)=\left(\bar{c}_{i}^{\prime}, p\right)=1$ for all $p \in E\left(C_{i}\right)$ and $1 \leq i \leq k$ and $\left(\bar{r}_{j}, p\right)=\left(\bar{r}_{j}^{\prime}, p\right)=1$ for all $p \in E(R)$ and $1 \leq j \leq n$ and units $u_{i}, u_{i}^{\prime} \in U\left(C_{i}\right), \hat{u}_{j}, \hat{u}_{j}^{\prime} \in U(R)$ we obtain that the automorphisms $\alpha_{1}=\operatorname{diag}\left(u_{1}, \cdots, u_{k}, \hat{u}_{1}, \cdots, \hat{u}_{n}\right)$ and $\alpha_{2}=\operatorname{diag}\left(u_{1}^{\prime}, \cdots, u_{k}^{\prime}, \hat{u}_{1}^{\prime}, \cdots, \hat{u}_{n}^{\prime}\right)$ satisfy $x \alpha_{1}=$ $\left(\bar{c}_{1}, \cdots, \bar{c}_{k}, \bar{r}_{1}, \cdots, \bar{r}_{n}\right)$ and $\left(\bar{c}_{1}^{\prime}, \cdots, \bar{c}_{k}^{\prime}, \bar{r}_{1}^{\prime}, \cdots, \bar{r}_{n}^{\prime}\right)=y \alpha_{2}$. Hence we may assume without loss of generality that $\operatorname{gcd}\left(c_{i}, p\right)=\operatorname{gcd}\left(c_{i}^{\prime}, p\right)=1$ for all $p \in E\left(C_{i}\right)(1 \leq i \leq k)$ and $\operatorname{gcd}\left(r_{j}, p\right)=$ $\operatorname{gcd}\left(r_{j}^{\prime}, p\right)=1$ for all $p \in E(R)(1 \leq j \leq n)$. Now let $d_{x}=\operatorname{gcd}\left(c_{1}, \cdots, c_{k}, r_{1}, \cdots, r_{n}\right)$ and $d_{y}=\operatorname{gcd}\left(c_{1}^{\prime}, \cdots, c_{k}^{\prime}, r_{1}^{\prime}, \cdots, r_{n}^{\prime}\right)$, then $d_{x}=d_{y}$ by what we have arranged so far and dividing by $d_{x}=d_{y}$ we may assume that $\operatorname{gcd}\left(c_{1}, \cdots, c_{k}, r_{1}, \cdots, r_{n}\right)=\operatorname{gcd}\left(c_{1}^{\prime}, \cdots, c_{k}^{\prime}, r_{1}^{\prime}, \cdots, r_{n}^{\prime}\right)=$ 1. Let $d=\operatorname{gcd}\left(r_{1}, \cdots, r_{n}\right)$ and $e=\operatorname{gcd}\left(r_{1}^{\prime}, \cdots, r_{n}^{\prime}\right)$, then the elements $\left(r_{1}, \cdots, r_{n}\right)$ and $(d, \cdots, d)$ have the same characteristic in $R^{n}$ which is transitive by Lemma 3.14 , hence there is an automorphism $\alpha_{x}$ of $R^{n}$ such that $\left(r_{1}, \cdots, r_{n}\right) \alpha_{x}=(d, \cdots, d)$. Similarly, $\left(r_{1}^{\prime}, \cdots, r_{n}^{\prime}\right) \alpha_{y}=(e, \cdots, e)$ for some automorphism $\alpha_{y}$ of $R^{n}$. Thus $i d_{c} \oplus \alpha_{x}$ and $i d_{c} \oplus \alpha_{y}$ are automorphisms of $C \oplus R^{n}$ sending $x$ onto $\left(c_{1}, \cdots, c_{k}, d, \cdots, d\right)$ and $y$ onto $\left(c_{1}^{\prime}, \cdots, c_{k}^{\prime}, e, \cdots, e\right)$ 
respectively. Therefore it remains to show that there exists an automorphism $\alpha$ of $C \oplus R_{n}$ sending $\left(c_{1}^{\prime}, \cdots, c_{k}^{\prime}, e, \cdots, e\right)$ onto $\left(c_{1}, \cdots, c_{k}, d, \cdots, d\right)$. Since $\operatorname{gcd}\left(c_{1}, \cdots, c_{k}, d\right)=1$ there are integers $l_{1}, \cdots, l_{k}$ and $l_{d}$ such that $\sum_{i \leq k} l_{i} c_{i}+l_{d} d=1$. Then the mapping

$$
\left(\begin{array}{cccccccccc}
1 & 0 & 0 & \cdots & 0 & l_{1} & 0 & \cdots & \cdots & 0 \\
0 & 1 & 0 & \cdots & 0 & l_{2} & 0 & \cdots & \cdots & 0 \\
\vdots & \vdots & \vdots & \vdots & \vdots & \vdots & \vdots & \vdots & \vdots & \vdots \\
0 & 0 & 0 & \cdots & 1 & l_{k} & 0 & \cdots & \cdots & 0 \\
0 & 0 & 0 & \cdots & 0 & \left(l_{d}-1\right) & 0 & \cdots & \cdots & 1 \\
0 & \cdots & \cdots & \cdots & 0 & 0 & \cdots & \cdots & 1 & 0 \\
\vdots & \vdots & \vdots & \vdots & \vdots & \vdots & \vdots & \vdots & \vdots & \vdots \\
0 & \cdots & \cdots & \cdots & 0 & 0 & 1 & \cdots & \cdots & 0 \\
0 & \cdots & \cdots & \cdots & 0 & 1 & 0 & \cdots & \cdots & 0
\end{array}\right)
$$

sends $\left(c_{1}, \cdots, c_{k}, d, \cdots, d\right)$ onto $\left(c_{1}, \cdots, c_{k}, 1, d, \cdots, d\right)$. However, this matrix may be reduced by elementary row operations to a block diagonal form $\left(\begin{array}{ll}I & 0 \\ 0 & D\end{array}\right)$ where $D$ is invertible and so the mapping is an automorphism. Similarly $\left(c_{1}^{\prime}, \cdots, c_{k}^{\prime}, e, \cdots, e\right)$ can be mapped onto $\left(c_{1}^{\prime}, \cdots, c_{k}^{\prime}, 1, e, \cdots, e\right)$ by an automorphism. But now $(1, d, \cdots, d)$ and $(1, e, \cdots, e)$ have the same characteristic in $R^{n}$ (which is transitive by Lemma 3.14), hence there is an automorphism $\beta$ of $R^{n}$ mapping $(1, d, \cdots, d)$ onto $(1, e, \cdots, e)$. Moreover, by assumption (using that $\left.\operatorname{Hom}\left(R^{n}, C\right)=0\right)$ there is an automorphism $\gamma$ of $C$ mapping $\left(c_{1}, \cdots, c_{k}\right)$ onto $\left(c_{1}^{\prime}, \cdots, c_{k}^{\prime}\right)$ and so $\gamma \oplus \beta$ maps $\left(c_{1}, \cdots, c_{k}, 1, d, \cdots, d\right)$ onto $\left(c_{1}^{\prime}, \cdots, c_{k}^{\prime}, 1, e, \cdots, e\right)$ and this finishes the proof.

As a corollary we obtain

Theorem 3.31 If $G$ is a separable group such that for any type $\tau \in T_{c r}(G)$ which is not minimal we have $r k\left(G(\tau) / G^{\sharp}(\tau)\right) \geq 2$, then $G$ is weakly transitive.

Proof. It suffices to assume that $G$ is completely decomposable of finite rank. We prove the claim by induction on $n=r k(G)$. If $n=1$, then the claim is true by Lemma 3.14 since $G$ is homogeneous. Assume $n \geq 2$. If there are two maximal types in $T_{c r}(G)$ then we finish as in the proof of Theorem 3.20 case (a). If there is just one maximal type we can apply Theorem 3.30 since by hypothesis $r k\left(G(\tau) / G^{\sharp}(\tau)\right) \geq 2$.

Corollary 3.32 The class of weakly transitive completely decomposable groups is not closed under taking direct summands.

Proof. Choose any weakly transitive completely decomposable group $C$ and let $R$ be a rational group which does not satisfy condition (ii) of Theorem 3.17. Then $D=C \oplus R$ is 
not weakly transitive by Theorem 3.29 . However, its square $D \oplus D$ is weakly transitive by Theorem 3.30.

Our final theorem shows that in many cases the square of a weakly transitive groups is again weakly transitive.

Theorem 3.33 Let $G$ be a torsion-free abelian group with endomorphism ring $R$ which is a principal ideal domain. If all endomorphisms of $G$ are monic, then $G \oplus G$ is weakly transitive.

Proof. Assume that we are given $0 \neq x, y \in G \oplus G$ and $x A=y, y B=x$ for some endomorphisms $A, B$ of $G \oplus G$. We may regard $A$ and $B$ as two by two matrices over $R$, hence by Grove (1983) Theorem 5.2 there are invertible matrices $P, Q, P^{\prime}, Q^{\prime} \in M_{2}(R)$ and diagonal matrices $D_{1}=\operatorname{diag}\left(d_{1}, d_{2}\right), D_{2}=\operatorname{diag}\left(d_{1}^{\prime}, d_{2}^{\prime}\right)$ such that $d_{1} \mid d_{2}$ and $d_{1}^{\prime} \mid d_{2}^{\prime}$ and $A=P D_{1} Q$ and $B=P^{\prime} D_{2} Q^{\prime} . D_{1}$ and $D_{2}$ are known as the Smith Normal Form of $A$ and $B$ respectively. Let $\tilde{x}=x P$ and $\tilde{y}=y P^{\prime}$, then it suffices to find an invertible matrix $T_{1}$ such that $\tilde{x} T_{1}=\tilde{y}$ since it then follows that $x P T_{1} P^{\prime-1}=y$ and $P T_{1} P^{\prime-1}$ is an automorphism of $G \oplus G$. By assumption we get $\tilde{x} D_{1} Q P^{\prime}=\tilde{y}$ and $\tilde{y} D_{2} Q P=\tilde{x}$. Let $S=Q P^{\prime}$ then $S$ is an automorphism of $G \oplus G$, so it suffices to find an invertible matrix $T_{2}$ such that $\tilde{x} T_{2}=\tilde{x} D_{1}$ (then $\tilde{x} T_{2} S=\tilde{y}$ and we are finished). Let $\tilde{x}=\left(x_{1}, x_{2}\right)$, hence $\tilde{x} D_{1}=\left(x_{1} d_{1}, x_{2} d_{2}\right)$. Without loss of generality we may assume that $\operatorname{gcd}\left(x_{1}, x_{2}\right)=1$. We have $\left(\tilde{x} D_{1}\right) S D_{2} Q P=\tilde{x}$ hence letting $W=S D_{2} Q P$ it follows that $\tilde{x} D_{1} W=\tilde{x}$. From now on we regard $G \oplus G$ as an $R$-module and note that group-homomorphisms are the same as $R$-homomorphisms since $R$ is commutative. Therefore, since $\chi(\tilde{x})=\chi\left(\tilde{x} D_{1}\right)$ (as elements of the $R$-module $G$ ) and $d_{1} \mid d_{2}$ we obtain that $d_{1}$ is a unit of $R$, without loss of generality $d_{1}=1$. Let $W=\left(\begin{array}{ll}a & b \\ c & e\end{array}\right)$ with $a, b, c, e \in R$. We obtain the following equations:

$$
x_{1} a+x_{2} d_{2} c=x_{1} \text { and } x_{1} b+x_{2} d_{2} e=x_{2} .
$$

Assume first that $x_{1}$ and $x_{2}$ are linearly independent elements in $G$ viewed as an right $R$ - module. Then $d_{2} c=0=b$ and $a=1=d_{2} e$, hence $d_{2}$ is an invertible element in $R$. Thus $D_{1}$ is already an automorphism and we may choose $T_{2}=D_{1}$. It remains to consider the case when $x_{1}$ and $x_{2}$ are dependent over $R$, i.e. $x_{1} r_{1}-x_{2} r_{2}=0$ for some $0 \neq r_{1}, r_{2} \in R$ with $\operatorname{gcd}\left(r_{1}, r_{2}\right)=1$. Since all endomorphisms of $G$ are monic it suffices to find an automorphism $\alpha$ sending $\left(x_{1}, x_{2}\right) r_{1}$ onto $\left(x_{1}, x_{2} d_{2}\right) r_{1}$. Then $\left(x_{1}, x_{2}\right) \in \operatorname{ker}\left(r_{1} \alpha-r_{1} D_{1}\right)$ and hence $r_{1}\left(\alpha-D_{1}\right)=0$ which implies that $D_{1}=\alpha$ is an automorphism. But $\left(x_{1}, x_{2}\right) r_{1}=$ $\left(x_{1} r_{1}, x_{2} r_{1}\right)=\left(x_{2} r_{2}, x_{2} r_{1}\right)$ and similarly, $\left(x_{1}, x_{2} d_{2}\right) r_{1}=\left(x_{2} r_{2}, x_{2} r_{1} d_{2}\right)$. Clearly $\chi\left(\left(r_{2}, r_{1}\right)\right)=$ $\chi\left(\left(r_{2}, r_{1} d_{2}\right)\right)$ since $\operatorname{gcd}\left(x_{1}, x_{2}\right)=\operatorname{gcd}\left(r_{1}, r_{2}\right)=1$ and so there is an automorphism of $R^{2}$ sending $\left(r_{2}, r_{1}\right)$ onto $\left(r_{2}, r_{1} d_{2}\right)$. Note that $R^{2}$ is transitive by an easy argument since pure submodules of finite rank are direct summands. Clearly this automorphism induces an 
automorphism of $G \oplus G$ that sends $\left(x_{1}, x_{2}\right) r_{1}$ onto $\left(x_{1}, x_{2} d_{2}\right) r_{1}$.

\section{REFERENCES}

Arnold, D. (1980). Finite rank torsion-free abelian groups and rings. Lecture Notes in Mathematics 931, Springer Verlag.

Carroll, D., Goldsmith, B. (1996) On transitive and fully transitive abelian $p$-groups. Proc. of the Royal Irish Academy 96A:33-41.

Corner, A.L.S. (1976) The independence of Kaplansky's notions of transitivity and full transitivity. Quart. J. Math. Oxford 27:15-20.

Corner, A.L.S., Göbel, R. (1985). Prescribing endomorphism algebras - a unified treatment. Proc. London Math. Soc. 50:447-479.

Dedekind, R., Dirichlet, R.G. (1968). Zahlentheorie. Chelsea Publishing Conmpany, New York.

Dobrusin, Yu. B. (1985). Extensions of partial endomorphisms of torsion-free abelian groups. Abelian groups and modules, Tomsk. Gos. Univ., Tomsk 36-59.

Dugas, M., Mader, A., Vinsonhaler, C. (1987) Large E-rings exist. J. of Algebra 108:88-101.

Dugas, M., Shelah, S. (1989). E-transitive groups in L. Abelian Group Theory, Amer. Math. Soc. 191-199.

Files, S. (1996). On transitive mixed abelian groups. Abelian Group Theory: Proceedings of the International Conference at Colorado Springs, Lecture Notes in Pure and Appl. Math. 182:243-251.

Files, S. (1997). Transitivity and Full Transitivity for Nontorsion modules. J. Algebra 197:468-478.

Files, S., Goldsmith, B. (1998). Transitive and fully transitive groups. Proc. Am. Math. Soc. 126:1605-1610.

Fuchs, L. (1970, 1973). Infinite Abelian Groups, Vol. I and II. Academic Press.

Goldsmith, B., Strüngmann, L. Torsion weakly transitive abelian grousp. in preparation.

Griffith, P. (1968). Transitive and fully transitive primary abelian groups. Pacific J. Math. 25:249-254.

Grinshpon, S. Ya. (1982). On the structure of fully characteristic subgroups of abelian torsion-free groups. Abelian groups and modules, Tomsk. Gos. Univ., Tomsk 56-92.

Grinshpon, S. Ya., Misyakov, V. M. (1991). Full transitivity of direct products of abelian groups. Abelian groups and modules, Tomsk. Gos. Univ., Tomsk 23-30.

Grinshpon, S. Ya., Misyakov, V. M. (1986). Fully transitive abelian groups', Abelian groups and modules, Tomsk. Gos. Univ., Tomsk 12-27.

Grove, L. (1983). Algebra, Pure and applied Mathematics, Academic Press 1983.

Hausen, J. (1987) E-transitive torsion-free abelian groups. J. of Algebra 107:17-27. 
Hennecke, G. (1999). Transitivitätseigenschaften abelscher p-Gruppen. doctoral thesis, Essen University.

Hennecke, G., Strüngmann, L. (2000). Transitivity and Full Transitivity for $p$-local Modules. Archiv der Mathematik 74:321-329.

Hill, P. (1969) On transitive and fully transitive primary groups. Proc. Amer. Math. Soc. 22:414-417.

Kaplansky, I. (1954, 1969). Infinite abelian groups, University of Michigan Press, Ann Arbor. Krylov, P. A. (1989). Strongly homogeneous torsion-free abelian groups. Sibirsk. Mat. Zh. $24: 77-84$.

Krylov, P. A. (1986). Irreducible abelian groups and their endomorphism rings. Abelian groups and modules, Tomsk. Gos. Univ., Tomsk 73-100.

Mader, A. (2000). Almost completely decomposable abelian groups, Algebra, Logic and Applications 13, Gordon and Breach.

Meehan, C., Strüngmann, L. Rational rings relaterd to weakly transitive torsion-free groups. submitted.

Megibben, C. (1966). Large subgroups and small homomorphisms. Michigan Mathematical Journal 13:153-160.

Misyakov, V. M. (1993). Full transitivity of reduced abelian groups. Abelian groups and modules, Tomsk. Gos. Univ., Tomsk 134-156.

Paras, A., Strüngmann, L. (2003). Fully transitive $p$-groups with finite first Ulm subgroup. Proc. Amer. Math. Soc., Proc. AMS 131:371-377. 